\title{
Do modelo tradicional ao ambiente virtual de ensino na rede pública e privada do estado de São Paulo
}

From traditional model to virtual teaching environment in the public and private network of the São Paulo state Del modelo tradicional al ambiente de enseñanza virtual en la red pública y privada del estado de São Paulo

\author{
Alex Moura Silva \\ Mestrando em Educação, Universidade Ibirapuera \\ Docente na SEDUC/SP e na Unicesumar \\ E-mail: alex.m.s.2018@gmail.com \\ ORCID: https://orcid.org/0000-0003-4106-7933 \\ Francisco Alexandre da Silva \\ Mestrando em Educação, Universidade Ibirapuera \\ Professor na SEDUC/SP \\ E-mail: franciscounib@gmail.com \\ ORCID: https://orcid.org/0000-0002-0922-2203
}

\begin{abstract}
RESUMO
Em consequência a pandemia causada pelo novo coronavírus, no Brasil e no mundo a educação básica passou por diversas transformações. O objetivo deste artigo é refletir sobre os impactos da pandemia causada pela COVID-19 e analisar os desafios da práxis docente da rede pública (Secretaria de Educação do Estado de São Paulo - SEDUC/SP, Secretaria Municipal de Educação de São Bernardo do Campo - SME/SBC) e particular do município de São Bernardo do Campo. De acordo com os procedimentos técnicos, o presente artigo caracteriza-se como uma revisão bibliográfica e estudo de caso, visto que, foram vinte artigos lidos, sendo utilizados doze artigos e, consequentemente, sete artigos descartados. Aplicou-se um questionário aos 125 docentes da educação básica da rede pública e privada do estado de São Paulo. Os resultados da pesquisa foram analisados através de tabela sobre o perfil do corpo docente e as expectativas dos mesmos em relação à formação de professores e o ensino remoto. Por fim, embora as Tecnologias de Informação e Comunicação (TICs) são presentes no século XXI, existem diversos fatores que acabam influenciando na relação práxis pedagógicas e TICs.
\end{abstract}

Palavras-chave: COVID-19, Educação básica, Práxis docente, TICs.

\begin{abstract}
As a result of the pandemic caused by the new coronavirus, in Brazil and in the world, basic education has undergone several transformations. The purpose of this article is to reflect on the impacts of the pandemic caused by COVID-19 and to analyze the challenges of teaching practice in the public network (Secretariat of Education of the State of São Paulo - SEDUC/SP, Municipal Department of Education of São Bernardo do Campo - SME/SBC) and private in the municipality of São Bernardo do Campo. According to the technical procedures, this article is characterized as a literature review and case study, since there were twenty articles read, twelve articles were used and, consequently, seven articles were discarded. A questionnaire was applied to 125 teachers of basic education from public and private schools in the state of São Paulo. The research results were analyzed through a table on the profile of the faculty and their expectations in relation to teacher training and remote teaching. Finally, although Information and Communication Technologies (ICTs) are present in the 21st century, there are several factors that end up influencing the relationship between pedagogical praxis and ICTs.
\end{abstract}

Keywords: COVID-19, Basic education, Teaching praxis, ICTs.

\section{RESUMEN}

Como resultado de la pandemia provocada por el nuevo coronavirus, en Brasil y en el mundo, la educación básica pasó por varias transformaciones. Este artículo tiene como objetivo reflexionar sobre los impactos de la pandemia provocada por el COVID-19 y analizar los desafíos de la práctica docente en la red pública (Secretaría de Educación del Estado de São Paulo - SEDUC/SP, Secretaría Municipal de Educación de São Bernardo do Campo - SME/SBC) y privada en el municipio de São Bernardo do Campo. De acuerdo con los procedimientos técnicos, este artículo se caracteriza como una revisión de literatura y estudio de caso, ya que se leyeron veinte artículos, se utilizaron doce artículos y, en consecuencia, se descartaron siete artículos. Se aplicó un cuestionario a 125 profesores de educación básica de escuelas públicas y privadas del estado de São Paulo. Los resultados de la investigación se analizaron a través de un cuadro sobre 
el perfil del profesorado y sus expectativas en relación a la formación docente y la enseñanza a distancia. Finalmente, si bien las Tecnologías de la Información y la Comunicación (TIC) están presentes en el siglo XXI, existen varios factores que acaban influyendo en la relación entre la praxis pedagógica y las TIC.

Palabras clave: COVID-19, Educación básica, Praxis docente, TIC.

\section{INTRODUÇÃO}

Primordialmente, na virada do ano de 2019 para 2020, a cidade chinesa de Wuhan e, posteriormente, outros países do mundo foram surpreendidos pela maior pandemia do século XXI, a partir do vírus Sars-CoV-2, mais conhecido mundialmente como COVID-19 (OLIVEIRA, 2020).

Logo depois a pandemia causada pela COVID-19, fez com que as instituições de ensino migrassem do ensino presencial tradicional para o modelo online. Os professores tiveram que aprender a como utilizar as Tecnologias de Informação e Comunicação (TICs), ou seja, utilizar as ferramentas de softwares, hardwares e meios de telecomunicação.

O ensino remoto emergencial tem sido utilizado por várias Secretarias de Educação de várias unidades federais (UF), com objetivo de manter a qualidade de ensino dos alunos em suas respectivas residências.

Até o século XX, o professor sempre foi visto como detentor único do conhecimento, porém com a chegada do novo milênio e posteriormente o fato inédito da pandemia da COVID-19, a educação brasileira passou por diversas transformações, uma delas foi o ensino humanizado, onde o professor passa a ser o mediador do conhecimento e o mesmo leva em consideração a subjetividade de cada indivíduo em sala de aula.

O conceito faz lembrar a escola quando não existia a pandemia, quando professores e alunos podiam se aglomerar em um espaço reduzido. Com a chegada da COVID-19 o ensino presencial passou para o ambiente virtual. Além disso, um dos pontos de destaque nessa temática é a deficiência na formação inicial dos professores em relação à utilização das TICs na prática pedagógica (OLIVEIRA, 2020).

O objetivo do presente artigo é compreender o processo de transição do ensino presencial para o ambiente virtual em tempos de pandemia da COVID-19. Utilizamos a metodologia estudo de caso, com a intenção de estudar a situação específica dos professores da Secretaria do Estado de São Paulo, da Secretaria Municipal de São Bernardo do Campo e das instituições de ensino particulares da região do ABC paulista.

Diante das questões apresentadas pelo momento atual, a presente pesquisa contempla três tópicos. O primeiro tópico aborda o perfil dos professores; o segundo tópico aborda a formação de professores e o terceiro e último tópico aborda o tema do Ensino Remoto.

\section{REFERENCIAL TEÓRICO}

\section{DA SALA DE AULA PARA O ENSINO REMOTO}

De acordo Correa (2000) o livro escolar era utilizado pelos professores e alunos como um guia para norteá-los no campo do conhecimento. Por outro lado, para Chartier (1990), os livros são instrumentos de educação que são utilizados como um projeto de formação social responsável pelo processo de ensino e aprendizagem desses indivíduos. 
Para Chartier (2010) o sistema educacional passou por diversas mutações até chegar ao processo de digitalização. Com as mudanças constantes na educação os meios de comunicação oral e escrita transformaram-se no processo de ensino e aprendizagem.

O século XXI exige da geração atual uma evolução constante, ou seja, vivemos em uma sociedade conectada e as Tecnologias de Informação e Comunicação (TICs) estão cada vez mais presentes em nosso cotidiano. Além disso, podemos perceber que as TICs estão presentes em todas as áreas da sociedade contemporânea como na educação. A cada dia que se passa, as TICs invadem a sala de aula tradicional, fazendo com que professores e alunos utilizem essas ferramentas como meio lúdico para o processo de ensino-aprendizagem (GUERRA et. al., 2020).

O Conselho Nacional de Educação (CNE/CP) publicou em 28 de abril de 2020 a CNE/CP $\mathrm{n}^{\circ} 005$, essa resolução trata sobre a reestruturação do calendário escolar e da readequação da carga horária das horas/aulas mínima anual que equivalem há 800 horas/aulas (CASTANHO et. al., 2020).

Os docentes passaram por um turbilhão de adaptações para contornarem as dificuldades no domínio tecnológico e posteriormente ensinar o alunado a utilizar as TICs. Após a pandemia, a tendência é oferecer para a área educacional um ensino híbrido para que a comunidade acadêmica esteja preparada para enfrentar as demandas do futuro, não somente no ambiente profissional, mas em outros imprevistos (GOMES et. al., 2020).

Debates atuais salientam que a pandemia causada pela COVID-19 acelerou o processo de inserção das TICs na educação básica, ou seja, a inclusão do ensino remoto era uma realidade próxima, porém ela não foi atingida, pela baixa adesão de aceitação do novo. O resultado dessa resistência foi a sobrecarga do corpo docente para elaborar os planos de aulas e acessar as plataformas digitais em tempo real ou offline, metodologias que já eram utilizadas pelas instituições de ensino superior antes da pandemia (OLIVEIRA, et. al., 2020).

Percebemos que o computador deixou de ser um acessório de auxílio do professor e transformou-se como exclusivo, isso fez com que as TICs ganhassem notoriedade no ambiente educacional (ANTONIASSI \& LACERDA, 2020). Além disso, a Secretaria de Educação do Estado de São Paulo, a Secretaria de Educação do Município de São Bernardo dos Campos e outras instituições de ensino privadas do $\mathrm{ABC} / \mathrm{Paulista}$ firmaram parcerias com a Microsoft Corporation, Google for Education, entre outras com objetivo de beneficiar a comunidade escolar.

Entretanto, a teoria de Jean Piaget afirma que é impossível o elemento cognitivo desenvolverse sem os elementos afetivos, bem como o inverso. Para Piaget, a afetividade possui um papel essencial no processo de aprendizagem do indivíduo. Portanto, o afeto é o elemento principal para desenvolver a cognição do indivíduo no processo de aprendizagem (DE OLIVEIRA et. al., 2021).

De acordo com o autor equatoriano Pérez (2022) o metaverso é um conceito que mescla o ambiente virtual e a realidade aumentada, como por exemplo o 3D Second Life, entre outras plataformas utilizadas como ferramenta de ensino na Educação Básica do Equador. Além disso, para o autor o metaverso é um caminho sem volta, ou seja, as TICs chegaram para ficar e fazer com que o processo de ensino-aprendizagem fique mais agradável.

Voltando para a nossa realidade, a pandemia obrigou a educação brasileira a apropriar-se das TICs de forma brusca como meio pedagógico no processo de ensino-aprendizagem (JUNIOR et. al., 2021). A expectativa é que após a pandemia as TICs serão agregadas no modelo de ensino híbrido.

\section{METODOLOGIA}

De acordo com a natureza metodológica, o presente artigo utilizou um estudo bibliográfico sobre ensino tradicional e ensino remoto na educação básica em período de pandemia do novo 
coronavírus (COVID-19). Para analisar a inserção das Tecnologias de Informação e Comunicação na educação tradicional brasileira utilizamos uma pesquisa bibliográfica e de campo. Para Severino (2017), a pesquisa bibliográfica caracteriza-se como um apanhado de dados utilizados em outros registros com análise documental de outros autores no ambiente científico.

Por outro lado, Severino (2017), define o estudo de caso como um objeto construído pelo autor da pesquisa através da práxis particular e, posteriormente, com objetivo de analisar os dados justificar a pesquisa de acordo com o referencial teórico de outros autores. Nesse sentido, de acordo com os procedimentos técnicos, a pesquisa caracteriza-se como um estudo de caso. Utilizamos a Plataforma Google Forms para analisar as respostas dos 125 professores da rede pública (Secretaria de Educação do Estado de São Paulo - SEDUC/SP, Secretaria Municipal de Educação de São Bernardo do Campo - SME/SBC) e particular do município de São Bernardo do Campo.

Do ponto de vista ético, cabe salientar que houve utilização de término de consentimento livre e esclarecido, mantendo o anonimato dos respondentes, sem nenhum tipo de conflito de interesses.

\section{RESULTADOS E DISCUSSÃO}

A presente pesquisa visou analisar o perfil dos docentes e o que eles responderam a respeito da formação de professores e sobre o conceito de Ensino Remoto.

De acordo com a Tabela 1, 78,4\% dos docentes são do gênero feminino, 18,4\% do gênero masculino e 2,2\% do gênero outros. Acrecentando-se que $86,4 \%$ são professores da Secretaria de Educação do Estado de São Paulo (SEDUC/SP), 6,6\% são professores da Secretaria Municipal do Município de São Bernardo do Campo (SME/SBC) e 6,4\% são professores de instituições privadas de ensino da educação básica.

Tabela 1 - Perfil dos Docentes

\begin{tabular}{|c|c|c|c|}
\hline Gênero & Fem. & Masc. & $\begin{array}{c}\text { Outros } \\
2,2 \%\end{array}$ \\
\hline \multirow{2}{*}{ Vínculo } & $78,4 \%$ & $18,4 \%$ & SEDUC-SP \\
& $86,4 \%$ & SME/SBC & $6,6 \%$ \\
& & $\begin{array}{c}\text { Particular } \\
6,4 \%\end{array}$ \\
\hline
\end{tabular}

Fonte: Elaborado pelos autores com base na pesquisa do ano de 2020.

De acordo com os dados apresentados na Figura 1, as palavras mais utilizadas para definir o conceito de formação de professores foram: conhecimento, empatia, atualização, aprendizagem, dedicação, comprometimento, tecnologia, resiliência. Apesar de algumas palavras apresentadas na Figura 1, resta compreender o significado delas no contexto. Podemos perceber que esses conceitos definidos pelos professores apontam aspectos do âmbito de conhecimento e atitudes.

Figura 1 - Nuvem de Palavras Relacionada à Formação de Professores 


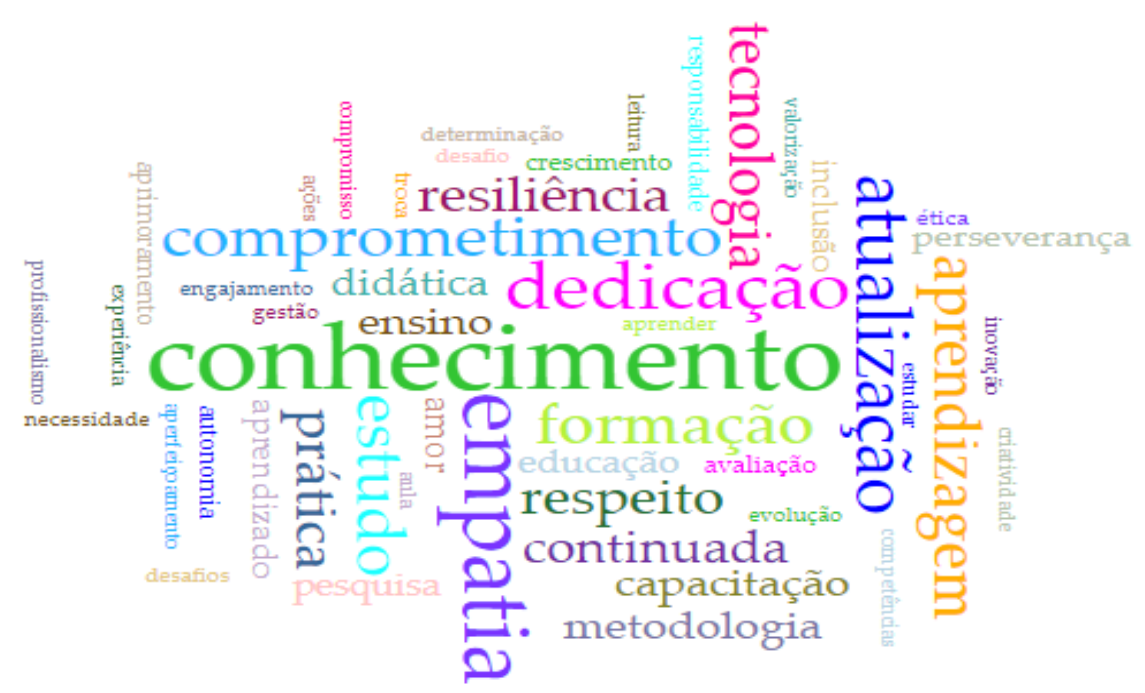

Fonte: voyant-tools.org (Adaptado pelos autores).

Sobre o Gráfico 1, as palavras que predominaram em relação ao Ensino Remoto são: tecnologia, dificuldade, empatia, disciplina, dedicação. Ou seja, por mais que a nossa sociedade encontra-se coberta pela bolha tecnológica, os professores não se sentem seguros para utilizar as tecnologias com domínio, ou seja, nesse aspecto os professores perceberam que precisam reaprender para ensinar os alunos nativos digitais.

Gráfico 1 - Palavras relacionadas ao Ensino Remoto

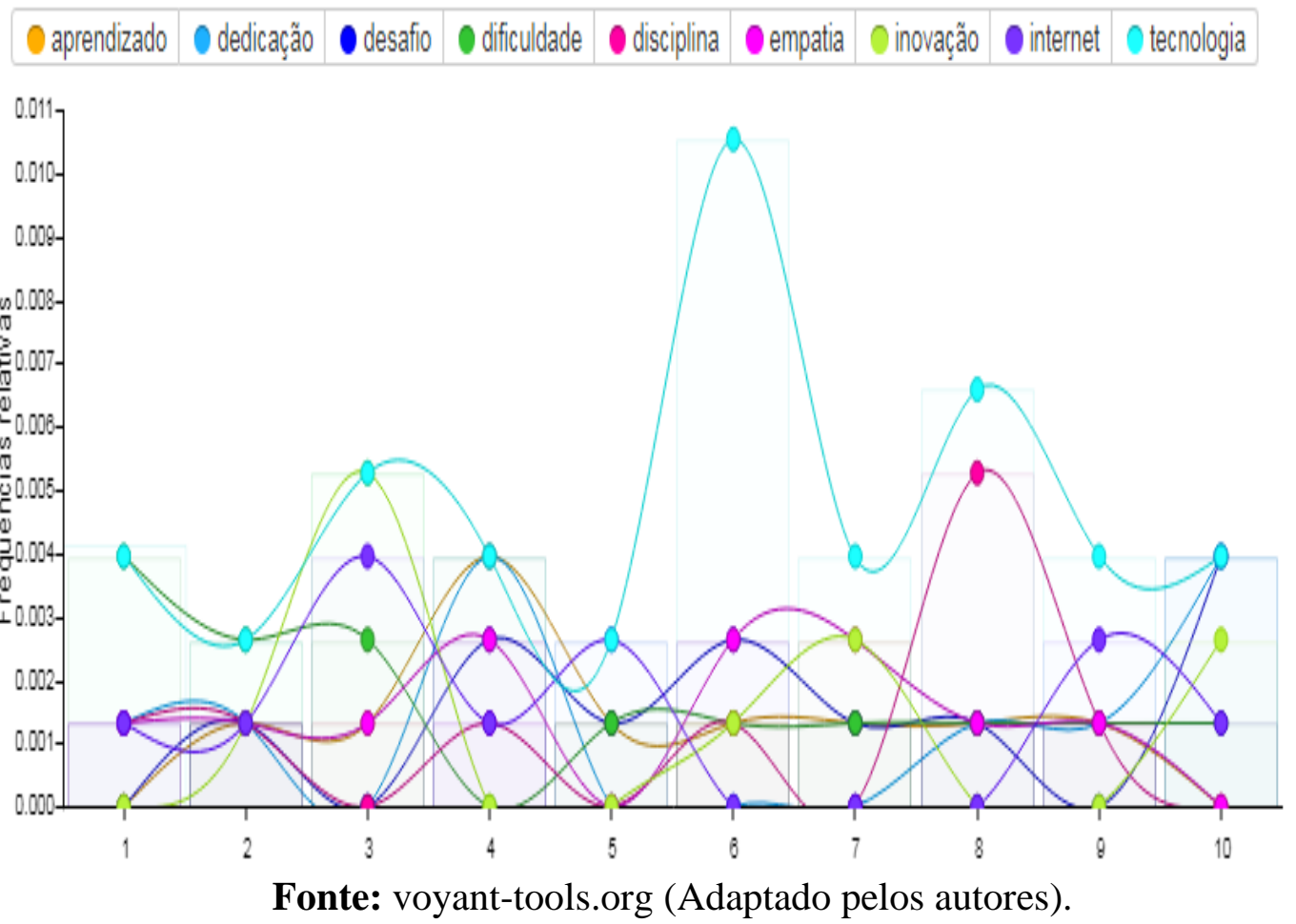




\section{CONSIDERAÇÕES FINAIS}

Em suma, o presente artigo abordou o processo de aceleração e inserção das Tecnologias de Comunicação e Informação (TICs) como ferramentas de ensino utilizadas pelos professores como práxis pedagógica e desenvolvimento no processo de ensino-aprendizagem dos alunos tanto em instituições de ensino públicas como em instituições privadas no Município de São Bernardo do Campo.

Os dados apresentados pelo Google Forms no estudo de caso realizado, permitiram perceber uma participação maior em relação ao gênero feminino $(78,4 \%)$, do que em relação ao sexo masculino $(18,4 \%)$. Ao observar o vínculo dos professores entrevistados, o que obteve maior número de participação foi a comunidade acadêmica da Secretaria da Educação do Estado de São Paulo - SEDUC/SP $(86,4 \%)$, levando em consideração que essa pasta possui um número maior de professores.

Em relação à definição da expressão formação de professores respondida pelos professores, algumas palavras apareceram numa menor escala, porém se destacaram, são elas: valorização, desafio, necessidade, perseverança, inclusão. Analisando essas respostas, podemos perceber que os professores sentem-se de certa forma, sobrecarregados com o sistema e cheios de desafios com as demandas do mercado e a subjetividade do seu alunado no cenário atual.

Quanto à definição da palavra Ensino Remoto respondida pelos professores, as palavras que se destacaram são: desafio, dificuldade, aprendizado, empatia, dedicação, tecnologia. Essas definições, só reforçam a realidade da educação brasileira, o corpo docente apresenta uma baixa aceitação na inserção das TICs como ferramenta de trabalho, porém ainda existe uma curiosidade para aprender a dominar as TICs e futuramente apropriar-se com fluência para ensinar os alunos em sala de aula.

De acordo com os dados levantados, podemos perceber as dificuldades apresentadas pelos professores em relação a utilização das TICs, a diferença de fluência das TICs em relação ao gênero do corpo docente, a distinção do choque cultura cultural partindo de uma visão tridimensional (universo escolar x professor x aluno) e a diferença na educação da rede pública e privada.

Finalizando, as obras de Chartier foram fundamentais para compreender a evolução das tecnologias, desde o modelo tradicional, por exemplo, o livro, até chegar às Tecnologias de Comunicação e Informação (TICs), como por exemplo, os hardwares (computadores, celulares, entre outros) e as plataformas digitais (Google Education, Microsoft, entre outros). Ou seja, essas ferramentas são fundamentais para o desenvolvimento do indivíduo na atualidade do século XXI.

A pandemia fez com que a educação brasileira acelerasse o processo de inserção das TICs no processo de ensino-aprendizagem dos alunos e formação dos professores. Com esse processo, será possível analisar futuramente a familiarização e formação docente com as TICs, o metaverso na educação do século XXI; o comportamento da "geração pandemia" no ambiente escolar; entre outras.

Por fim, podemos perceber que as TICs estão cada vez mais presentes em nosso cotidiano, porém as relações humanas são indispensáveis. As tecnologias servem como ferramenta de auxílio no processo de ensino aprendizagem, porém elas não substituem o papel do professor em sala de aula ou ambientes virtuais. 


\section{REFERÊNCIAS}

CANI, J. B. et al. Educação e covid-19: a arte de reinventar a escola mediando a aprendizagem "prioritariamente" pelas TDIC. Revista Ifes Ciência, v. 6, n. 1, p. 23-39, 2020.

CASTANHO, M. E. et al. A dimensão socioafetiva em ambiente virtual de aprendizagem nos tempos de Covid-19. 2020.

CHARTIER, Roger. Escutar os mortos com os olhos. Estudos avançados, v. 24, n. 69, p. 6-30, 2010.

CORRÊA, Rosa Lydia Teixeira. O livro escolar como fonte de pesquisa em História da Educação. Cadernos Cedes, v. 20, p. 11-23, 2000.

DE OLIVEIRA, A. F. T. et al. Da sala de aula às aulas remotas: um percurso cognitivo-afetivo em época de pandemia. Brazilian Journal of Development, v. 7, n. 2, p. 18718-18732, 2021.

GOMES, M. A. et al. Contexto atual do ensino remoto em tempos de covid-19: um estudo de caso com estudantes do ensino técnico. Brazilian Journal of Development, v. 6, n. 10, p. 79175-79192, 2020.

Guerra, M. das, Gomes, C., \& Ribeiro, W. (2020, junho 4). Sala de Aula Digital e o uso das Novas Tecnologias na Educação: Diálogos Interdisciplinares, 9(5), 36-49. Disponível em:

<https://revistas.brazcubas.br/index.php/dialogos/article/view/946>. Acesso em: 15 jul. 2021.

JUNIOR, C. A. et al. Ambiente Virtual De Aprendizagem: Importância Das Habilidades Tecnológicas Em Tempos De Pandemia Da Covid-19. Brazilian Journal of Development, v. 7, n. 2, p. 16849-16859, 2021.

LACERDA, M. F. L.; ANTONIASSI, A. M. Da Sala de Aula ao Ambiente Virtual: Adaptação dos/as Professores/as na Educação Infantil Frente ao Cenário Da COVID-19. Anais do CIET: EnPED: 2020 - (Congresso Internacional de Educação e Tecnologias | Encontro de Pesquisadores em Educação a Distância), São Carlos, ago. 2020. ISSN 2316-8722. Disponível em: <https://cietenped.ufscar.br/submissao/index.php/2020/article/view/1105>. Acesso em: 13 jul. 2021

MIRANDA, A. C. C. et al. Ambiente Virtual de Aprendizagem: Percepção Docente quanto à Expectativa de Desempenho. In: Congresso sobre Tecnologias na Educação (CTRL+E), 5. , 2020, Evento Online. Anais [...]. Porto Alegre: Sociedade Brasileira de Computação, 2020 . p. 326-335. Disponível em: <https://doi.org/10.5753/ctrle.2020.11410>. Acesso em: 18 jul. 2021.

OLIVEIRA, J. de F. A. C. et al. (2020). Educação no contexto da pandemia da Covid-19: adversidades e possibilidades. Itinerarius Reflectionis, 16(1), 01-17. Disponível em: 〈https://doi.org/10.5216/rir.v16i1.65332>. Acesso em: 15 jul. 2021.

OLIVEIRA, V. H. N. "O antes, o agora e o depois": alguns desafios para a educação básica frente à pandemia de COVID-19. Boletim de Conjuntura (BOCA), v. 3, n. 9, p. 19-25, 2020.

PÉREZ, Xavier Oswaldo Pacheco; CRESPO, Evelin Johanna Rosales. TICs en la educación en contextos de disrupción tecnológica. RECIAMUC, v. 6, n. 1, p. 139-148, 2022.

SANTOS JUNIOR, V. B. DOS; MONTEIRO, J. C. DA S. Educação e COVID-19: as Tecnologias Digitais Mediando a Aprendizagem em Tempos de Pandemia. Revista Encantar - Educação, Cultura e Sociedade, v. 2, p. 01-15, 15 maio 2020 .

SEVERINO, Antônio Joaquim. Metodologia do trabalho científico. Cortez editora, 2017. 